\title{
A GENERAL PROPERTY OF RANDOM WALK
}

\author{
BY H. KESTEN, ${ }^{1}$ D. ORNSTEIN AND F. SPITZER ${ }^{1}$
}

Communicated by J. L. Doob, June 6, 1962

Let $P(x, y)=P(0, y-x)$ be the transition function of a random walk on the set of lattice points $R=\{x: x$ has integer valued coordinates $\}$ in $d$-dimensional Euclidean space, $d \geqq 1$. Thus $0 \leqq P(0, x)$, $\sum_{x \in R} P(0, x)=1$ and it is also assumed that the additive group generated by $\{x: P(0, x)>0\}$ is $R$ itself. Probabilities of events concerning the resulting Markov process (random walk) $x_{n}, n \geqq 0$, starting at the point $x_{0}=x$ are denoted by $P_{x}[\cdot]$, in particular $P_{x}\left[x_{n}=y\right]$ $=P_{n}(x, y)$, the $n$th iterate over $R$ of the convolution operator $P(x, y)$.

If the random variable $T$ is the time of the first visit of the random walk to the origin after time 0 , then $r_{n}=P_{0}[T>n]$, which may also be defined analytically by

$$
1=\sum_{k=0}^{n} P_{k}(0,0) r_{n-k}, \quad n \geqq 0,
$$

has the property

$$
\lim _{n \rightarrow \infty} \frac{r_{n+1}}{r_{n}}=1 .
$$

Equation (1) which was proved independently, and by different methods, by two of the authors is the crucial lemma in the proof of the

TheOREM. For $x \neq 0$

$$
\lim _{n \rightarrow \infty} \frac{P_{x}[T>n]}{P_{0}[T>n]}=a(x)
$$

where $a(x)=\sum_{k=0}^{\infty}\left[P_{k}(0,0)-P_{k}(x, 0)\right]$, which converges for all $x$ in $R$ according to [3].

Both (1) and (2) are trivial in case the random walk is transient. We give a proof of (2) for every recurrent random walk, with the exception of the case when $d=1$ and $\sigma^{2}=\sum x^{2} P(0, x)<\infty$. (In this case equation (3) below is false, but more direct methods are available.)

Let $Q(x, y)$ be $P(x, y)$ with $x$ and $y$ restricted to $R-\{0\}$, with

${ }^{1}$ With partial support from NSF contract G 18837. 
$Q_{n}(x, y)$ the iterates of $Q$ over $R-\{0\}$. For $x, y \neq 0$

$$
g(x, y)=\sum_{n=0}^{\infty} Q_{n}(x, y)<\infty
$$

is the expected number of visits of $x_{n}$ to $y$ before $T$ if $x_{0}=x$. From [3] we need the result that

$$
\lim _{|y| \rightarrow \infty} g(x, y)=a(x) .
$$

For $x \neq 0$

$$
\begin{aligned}
P_{x}[T>n] & =\sum_{k=n}^{\infty} P_{x}[T=k+1]=\sum_{k=n}^{\infty} \sum_{y \neq 0} Q_{k}(x, y) P(y, 0) \\
& =\sum_{t \neq 0} g(x, t) \sum_{y \neq 0} Q_{n}(t, y) P(y, 0) .
\end{aligned}
$$

Setting

$$
\begin{gathered}
v_{n}(t)=\frac{\sum_{y \neq 0} Q_{n}(t, y) P(y, 0)}{P_{0}[T>n+1]}, \quad t \neq 0, \\
\frac{P_{x}[T>n]}{P_{0}[T>n]}=\frac{r_{n+1}}{r_{n}} \sum_{t \neq 0} g(x, t) v_{n}(t) .
\end{gathered}
$$

Using (1) and (3) the proof can be completed by an obvious compactness argument if we show that

$$
\begin{array}{ll}
\sum_{t \neq 0} v_{n}(t)=1 & \text { for } n \geqq 1, \\
\lim _{n \rightarrow \infty} v_{n}(t)=0 & \text { for each } t \neq 0 .
\end{array}
$$

Letting $y_{n}$ be the "reversed" random walk with transition function $P^{*}(x, y)=P(y, x), T^{*}$ the first visit of $y_{n}$ to the origin after time 0 , one has

$$
v_{n-1}(t)=P_{0}\left[y_{n}=t \mid T^{*}>n\right]
$$

so that each $v_{n}$ is a probability measure on $R-\{0\}$. Finally, selecting an integer $m$ such that $P_{m}^{*}(t, 0)>0$,

$$
v_{n-1}(t) P_{m}^{*}(t, 0) \leqq \frac{P_{0}\left[n<T^{*} \leqq n+m\right]}{P_{0}\left[T^{*}>n\right]}=\frac{r_{n}-r_{n+m}}{r_{n}}
$$

so that (1) implies that $v_{n}(t) \rightarrow 0$.

Hunt [1] first obtained a theorem analogous to (2) for two dimen- 
sional Brownian motion, and Kac [2] for a restricted class of random walks.

\section{REFERENCES}

1. G. Hunt, Some theorems on Brownian motion, Trans. Amer. Math. Soc. 81 (1956), 294-319.

2. M. Kac, A class of limit theorems, Trans. Amer. Math. Soc. 84 (1957), 459-471.

3. F. Spitzer, Hitting probabilities, J. Math. Mech. 11 (1962), 593-614.

CORNELL UNIVERSITY AND

STANFORD UNIVERSITY

\section{CLASSIFICATION OF $A E A$ FORMULAS BY LETTER ATOMS}

BY BURTON DREBEN, A. S. KAHR, AND HAO WANG

Communicated by A. M. Gleason, May 11, 1962

The class $G$ of all closed prenex schemata in the form $A x E u A y M x u y$ whose quantifier-free matrices $M x u y$ contain only dyadic schematic letters was shown in [2] to be a reduction class for quantification theory. Here we shall study the decision problems of various subclasses of this unsolvable class. Since a dyadic schematic letter may be followed by $x x, x u, u x, u u, y y, x y, y x, u y$, or $y u$, any letter atom occurring in a given matrix $M x u y$ is in one of these nine letter atomic forms. The subclasses of $G$ to be studied will be specified in terms of these forms.

Consider the four letter atomic forms $x y, y x, u y, y u$. First take any three of them. From [2] we know that any subclass of $G$ which includes all schemata whose letter atoms are in just these three forms is a reduction class and hence is unsolvable. Now take any two of the four forms. Combining them with the other five forms yields a subclass of $G$. In this way we obtain six subclasses of $G$ which divide into three pairs: $J=\{x y, u y\}, J^{*}=\{y x, y u\}, L=\{x y, y x\}, L^{*}=\{u y, y u\}$, $Q=\{x y, y u\}, Q^{*}=\{y x, u y\}$. We discuss these three pairs of classes in turn.

The subclasses $J$ and $J^{*}$ are solvable and contain axioms of infinity, i.e., contain some schemata having just infinite models. This is significant because thus far no naturally specifiable infinite class of schemata containing axioms of infinity has been shown solvable. Since 